\title{
Time-Resolved Fluorescence Microscopy Combined to Scanning ElectroChemical Microscopy: A New Way to Visualize Photo-Induced Electron Transfer Quenching with An Electrofluorochromic Probe
}

Laetitia Guerret-Legras, Jean-Frédéric Audibert, Indra M. Gonzalez-Ojeda, Galina V. Dubacheva, Gilles Clavier and Fabien Miomandre* Université Paris-Saclay, ENS Paris-Saclay, CNRS, PPSM, 4 avenue des Sciences, 91190 Gif/Yvette, France

*mioman@ens-paris-saclay.fr, 33-181875595

Supporting information 


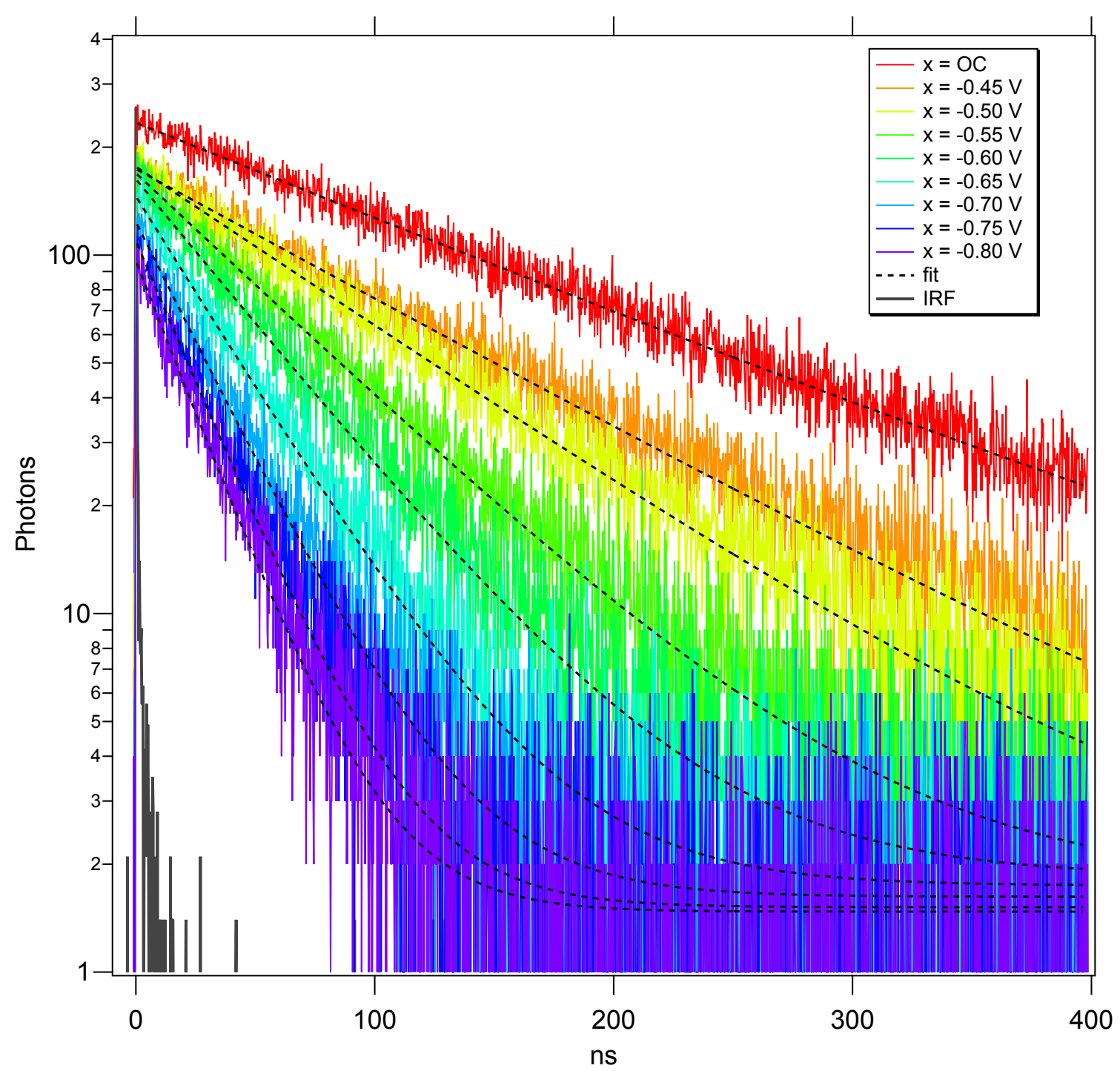

Figure S1: Fluorescence decays at various tip potentials. Red line is obtained in absence of electrochemical reaction (Open Circuit). Dark line is the Instrumental Response Function (IRF) in solvent only. Dashed lines are fitting by Levenberg -Marquardt method with minimization of $\chi^{2}$ from monoexponential decay equations. Reduced- $\chi^{2}$ span between 1.012 and 1.233 for the useful signal in the time window range. 

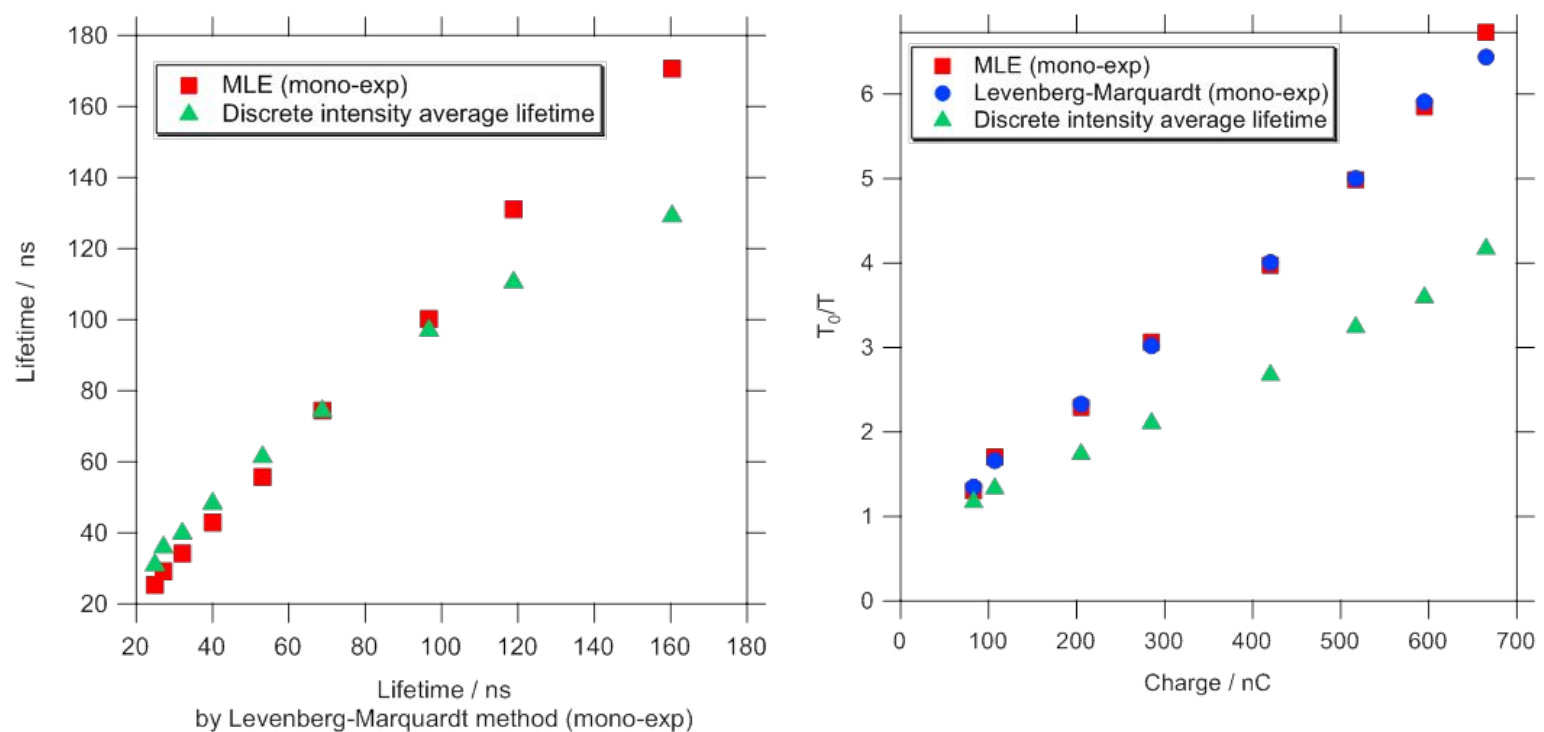

Figure S2: Comparison of lifetime estimation by different methods for simple exponential decay using Levenberg-Marquardt method (left). Maximum-Likelihood Estimator (red) and discrete intensity average lifetime (green). Deviation from linearity of the discrete intensity average lifetime for long lifetimes is due to incomplete decays in the time window range. Lifetime ratios vs. charge (right) on glass substrate for the different methods. MLE (red), Levenberg-Marquardt (blue) and discrete intensity average lifetime (green). 